\title{
Major Pelvic Bleeding Following a Stapled Transanal Rectal Resection: Use of Laparoscopy as a Diagnostic Tool
}

\author{
Giovanni Domenico Tebala, Abdul Qayyum Khan, Sean Keane \\ Department of Surgery, Colorectal Team, Noble's Hospital, Douglas, Isle of Man
}

Stapled transanal rectal resection (STARR) and stapled hemorrhoidopexy (SH) are well-established techniques for treating rectal prolapse and obstructed defecation syndrome (ODS). Occasionally, they can be associated with severe complications. We describe the case of a 59-year-old woman who underwent STARR for ODS and developed a postoperative pelvic hemorrhage. A computed tomography (CT) scan revealed a vast pelvic, retroperitoneal hematoma and free gas in the abdomen. Laparoscopy ruled out any bowel lesions, but identified a hematoma of the pelvis. Flexible sigmoidoscopy showed a small leakage of the rectal suture. The patient was treated conservatively and recovered completely. Surgeons performing STARR and SH must be aware of the risk of this rare, but severe, complication. If the patient is not progressing after a STARR or SH, a CT scan can be indicated to rule out intra-abdominal and pelvic hemorrhage. Laparoscopy is a diagnostic tool and should be associated with intraluminal exploration with flexible sigmoidoscopy.

Keywords: Rectal prolapse; Laparoscopy; Postoperative hemorrhage

\section{INTRODUCTION}

The use of staplers for the treatment of hemorrhoids and obstructed defecation syndrome (ODS) is a well-established approach that is accepted worldwide $[1,2]$ and that has several proven advantages with respect to other techniques. Stapled hemorrhoidopexy $(\mathrm{SH})$ and stapled transanal rectal resection (STARR) have changed our view of treatments for patients with hemorrhoids and/or rectal prolapse, but these techniques have the potential for serious complications. Herein, we report the case of a patient who experienced significant pelvic bleeding after a STARR.

\section{CASE REPORT}

A 50-year-old woman was referred to the colorectal surgery clinic

Received: July 15, 2016 - Accepted: August 13, 2016

Correspondence to: Giovanni D. Tebala, M.D.

Department of Surgery, Gastrointestinal Surgery Unit, Noble's Hospital,

Strang - Douglas, Isle of Man IM4 4RJ

Tel: +44-1624-650207, Fax: +44-1624-650183

E-mail:Giovanni.Tebala@gov.im

(C) 2016 The Korean Society of Coloproctology

This is an open-access article distributed under the terms of the Creative Commons Attribution NonCommercial License (http://creativecommons.org/licenses/by-nc/4.0) which permits unrestricted noncommercial use, distribution, and reproduction in any medium, provided the original work is properly cited. for abdominal discomfort and chronic constipation. She had a past history of pelvic endometriosis. At consultation, she complained of chronic constipation, abdominal and perineal discomfort, and incomplete evacuation. She had to spend a long time on the toilet and strain intensely before opening her bowel. Frequently, she had to push on the posterior wall of her vagina or use her finger to empty her rectum. She had to use daily laxatives and occasional enemas; nonetheless, she could open her bowel less than once a week. Our initial impression was ODS. In fact, her Longo's score was 22 , and her symptoms severity score was 22 as well.

At physical examination she had perineal descent with no external rectal prolapse and no external hemorrhoids, but she had rectoanal intussusception, a redundant anterior mucosa and a $5-\mathrm{cm}$ anterior rectocele. Her sphincter tone was fine, and her squeezing pressure was slightly reduced. Magnetic resonance imaging confirmed perineal descent and a rectocele. Endoanal ultrasound found a small anterior sphincteric tear, probably due to obstetric trauma. Rectal manometry was normal. With the diagnosis of ODS, she was referred for pelvic physiotherapy. After 6 months of rehabilitation, she had some slight, subjective improvement, but her Longo's score was still 22. To rule out a recurrence of her endometriosis or any other pelvic disease, she had diagnostic laparoscopy, which was completely negative.

A STARR procedure was offered and accepted. This was straightforward, with no intraoperative complications. At the end of the 


\section{$\begin{array}{cl}\text { Annals of } & \begin{array}{l}\text { Major Pelvic Ble } \\ \text { Diagnostic Tool }\end{array}\end{array}$}

\section{Coloproctology Giovanni Domenico Tebala, et al.}

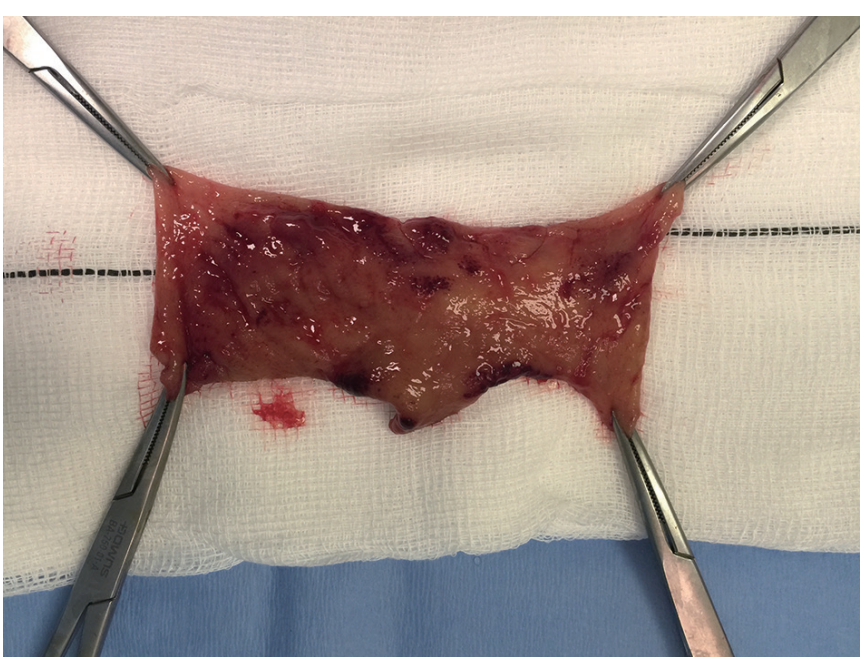

Fig. 1. Rectal cylinder removed by using a stapled transanal rectal resection.

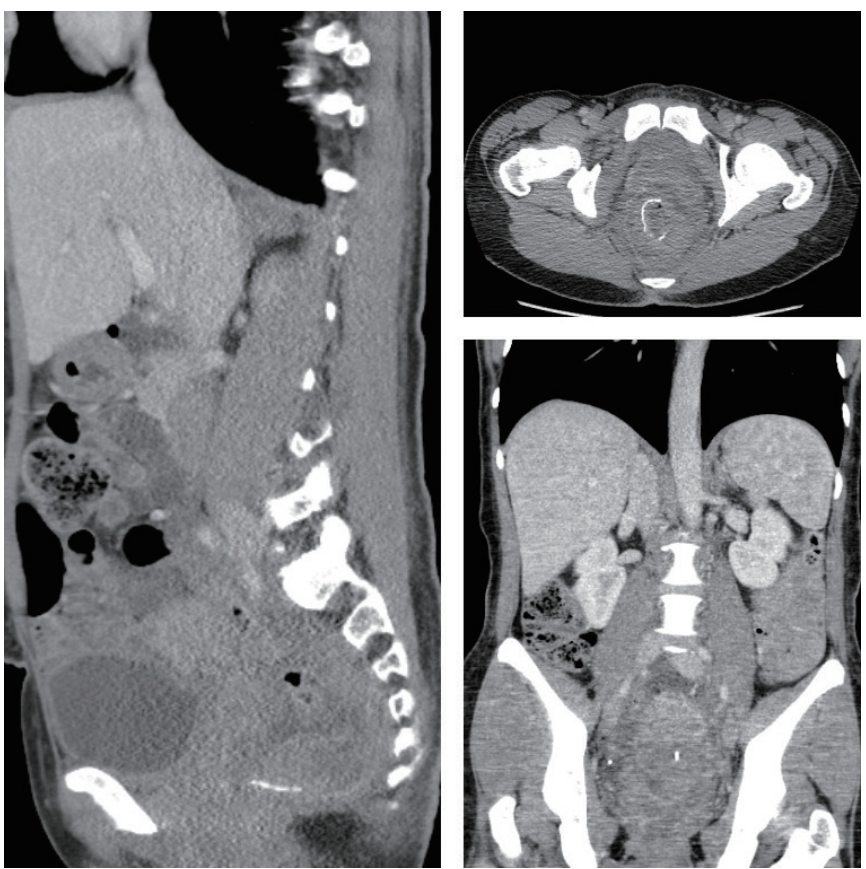

Fig. 2. Computed tomography scan showing a vast pelvic, retroperitoneal hematoma with no active bleeding.

surgery, the rectal suture was $5 \mathrm{~cm}$ above the dentate line and was complete. No bleeding points were noted. A $5-\mathrm{cm}$ prolapse was removed (Fig. 1). An anal absorbable tampon with a Foley catheter was left into her rectum, as usual. During the first night after the surgery, she had minimal rectal bleeding, which continued over the first postoperative day. She had some perineal and pelvic discomfort and was quite pale and tachycardic. Blood tests on postoperative day 2 showed a significant drop in her hemoglobin

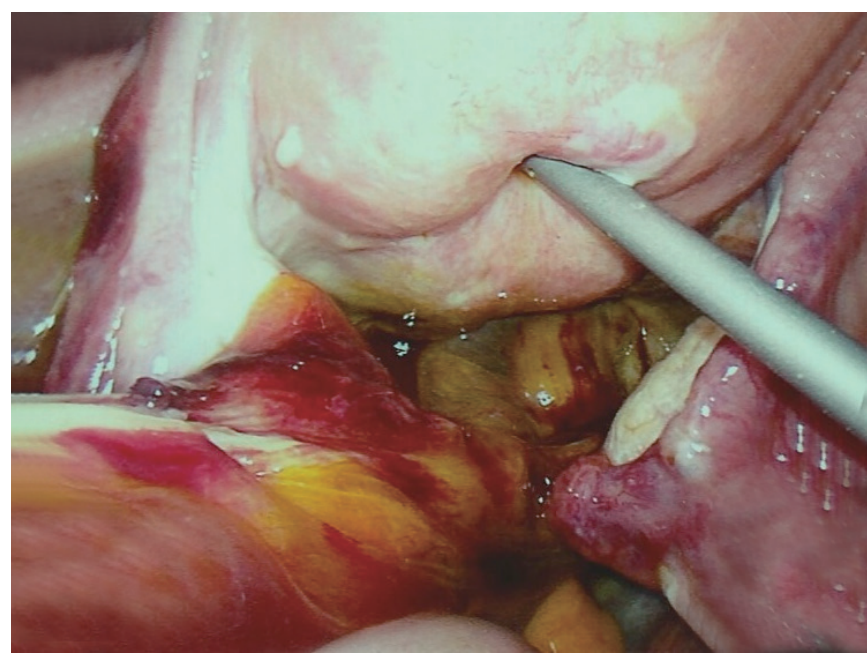

Fig. 3. Laparoscopic view of the pelvic hematoma. No intraperitoneal bleeding is seen.

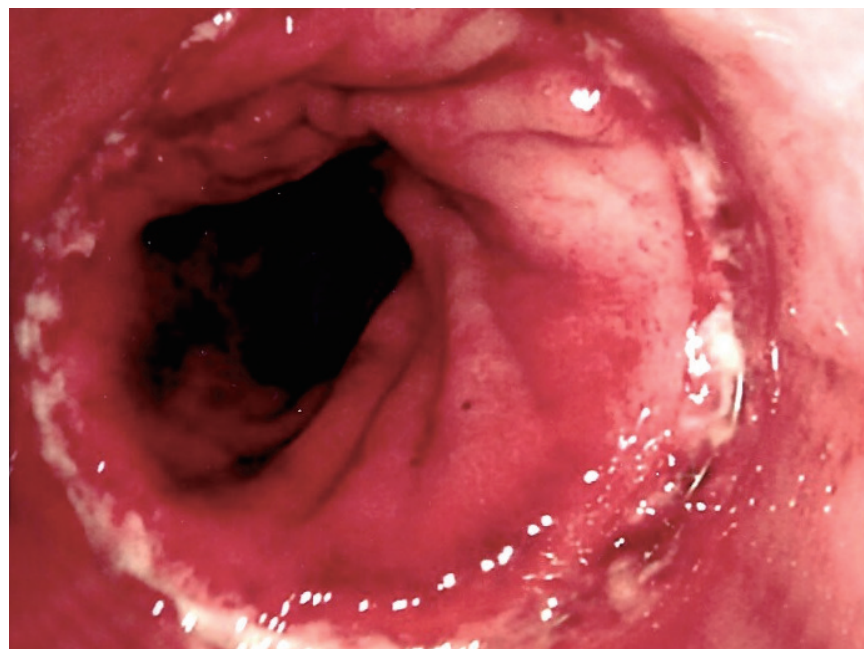

Fig. 4. Endoscopic view of the suture line with a minimal partial disruption of the suture, which might have allowed a small leakage of air towards the peritoneal cavity.

levels from 147 to $88 \mathrm{~g} / \mathrm{L}$. Fluid resuscitation was started, and an urgent abdominal and pelvic CT scan showed a huge extraperitoneal and retroperitoneal pelvic hematoma. No active arterial bleeding was demonstrated at the arterial phase. The anastomotic ring was complete, but a small amount of free gas in the peritoneal cavity was noted just above the bladder (Fig. 2). A diagnostic laparoscopy revealed no free gas, free fluid, blood or enteric contamination in the abdomen and no sign of peritoneal inflammation; only a vast extraperitoneal hematoma of the pelvis tracking the mesorectum and mesosigmoid up to the mid sigmoid and the lateral pelvic walls was found (Fig. 3). A flexible sigmoidoscopy showed a very small breakdown of the rectal suture (Fig. 4), 
which was closed with endoclips.

The patient responded well to conservative treatment with transfusions and laxatives, although she complained of perineal pain for more than 2 weeks. She was always clinically stable and nonseptic. She was discharged in good conditions 28 days after the first operation. At the 30-day postdischarge follow-up, the patient was completely pain free, and her Longo's score was back to 14 .

\section{DISCUSSION}

The SH and the STARR were proposed by Antonio Longo in the late '90s [1,2], following studies on the pathophysiology of the socalled hemorrhoidal disease and ODS. The initial crucial role of Longo's SH was to change our view of the so-called 'hemorrhoidal disease, demonstrating that what we call 'hemorrhoid' is only an epiphenomenon of a mucosal or full-thickness rectal prolapse, ratifying the clinical divorce between piles and other venous disorders, such as leg varices, which do not share the same pathophysiology. Longo's technique refocused our attention on the need both to avoid excision of the anorectal skin and to concentrate our therapeutic actions on the rectal mucosa above the dentate line. The SH has been endorsed by authoritative guidelines [3].

Longo's technique underwent evolutionary modifications derived mostly from demonstrations that a rectal prolapse is usually part of a complex pelvic floor failure as it is often associated with abnormalities of the anterior and the median compartments, mostly in women, and their consequences, such as rectoceles and cystoceles. A rectal prolapse has several degrees of severity and can be associated with anatomical and physiological abnormalities, such as rectoanal intussusception, perineal descent, and abnormal spasm of the puborectalis muscle. Pelvic floor failure with a rectal prolapse and/or a rectocele is the cause of ODS.

The STARR is an accepted treatment for patients with ODS and with a rectal prolapse [4]. The theoretical difference between the SH and the STARR is in the thickness of the tissue removed, being only mucosa and submucosa in the SH and the full thickness in the STARR. However, finding muscular, and even adipose, tissue in the specimens from the $\mathrm{SH}$ is a common experience. The different traction technique used in the STARR can account for the wider specimen with respect to the $\mathrm{SH}$. As hemorrhoidal disease is a consequence of a rectal prolapse, the STARR is increasingly being performed for the treatment of patients with hemorrhoids. In fact, the excision of a rectal prolapse will reduce the hemorrhoidal tissue back into the anal canal, thus re-establishing the correct anorectal anatomy, which is the core of Longo's theories.

Although every surgical procedure is well known to bear the risk of complications, surprisingly, during the last 10 years, the international surgical community has targeted the quite few, but major, complications of the SH and the STARR $[5,6]$. However, a recent metanalysis documented that the rate of complications in "conventional" hemorrhoidectomies was higher than it was in the SH [7]. The European, German, and Italian STARR Registries re- ported an incidence of bleeding from $2.9 \%$ to $5 \%$ after this procedure [8-10]; however, very few cases of massive perirectal bleeding have been reported so far [11-14], and only one case after a STARR has been reported [14].

Bleeding after a STARR or a SH can be intra or extrarectal. Intrarectal external bleeding is quite easy to identify and is usually self-resolving. It often comes from the venous hemorrhoidal plexus or from peripheral branches of one of the hemorrhoidal arteries. On the contrary, a massive extrarectal bleeding is not easy to diagnose as the only initial clinical sign may be a hemodynamic derangement in a patient who is not progressing well after surgery. Blood from an injured major arterial branch can collect within the ischiorectal fossa and track up following the mesorectum and mesosigmoid beyond the limits of the pelvis up to the retroperitoneum. Theoretically, this is a big space in which a huge amount of blood can collect, causing massive life-threatening hypovolaemia. Fortunately, the increased pressure within the pelvis due to the expanding hematoma overcomes the bleeding pressure and can ultimately manage to stop the bleeding. If bleeding is suspected, an arterial-phase CT scan is mandatory to confirm the presence of the hematoma and rule out an active bleeding point. In our case, the CT scan showed a vast pelvic and retroperitoneal hematoma, but no contrast leak.

Unfortunately, in our case, CT also showed a small quantity of intraperitoneal gas. Even though the CT scan showed some tiny bubbles of gas tracking from the rectal suture anteriorly to the Retzius space, thus suggesting an air leakage from the rectum, we had to rule out the much more relevant event of a small bowel injury due to entrapment of a small bowel loop in the stapled suture. However, in that case, we would have expected a greater amount of free air on the CT scan and a clinical picture of acute peritonitis. Fortunately, neither was present, and laparoscopic exploration produced no evidence of any bowel perforation.

The most likely mechanism of injury in this case was, in our opinion, a defective closure by the stapling device that failed to produce a satisfactory hemostasis and that caused both arterial bleeding from a perirectal branch of the inferior rectal artery and a minimal air leak from the suture. Probably this was due to excessive tissue on one side of the suture. Another possibility could be that a primary perianastomotic hematoma had caused a secondary partial disruption of the suture, as described by some authors [14].

The treatment of postoperative bleeding after a STARR should usually start with conservative measures, provided the patient's clinical conditions are stable and no sepsis is present, as in our case $[9,12]$. A reoperation may be required, but often only to get a precise diagnosis. A more aggressive approach may even require ligation of the internal iliac arteries or a low anterior resection [5]. We believe a simple embolization of the bleeding vessel would have been enough in our case if conservative treatment had failed. We agree with other authors [14] that laparoscopy with simultaneous anorectal exploration with flexible endoscopy is the best in- 
vestigative procedure to be performed in the event of massive bleeding and hematoma after a STARR or a SH. This approach would also allow drainage of the hematoma transabdominally, transrectally or transperineally, if indicated. In our case, this was not considered due to the absence of either sepsis or an expanding hematoma. Some authors prefer to open the peritoneal pelvic reflection and remove the hematoma laparoscopically. While appreciating that this maneuver can be effective in reducing the risk of sepsis, our fear was that it would have reduced the hemostatic effect of increased pelvic pressure on the bleeding vessels, so in our case, we preferred to leave the "natural pelvic packing" represented by the hematoma itself. Simultaneous anorectal exploration is mandatory in order to be able to check the suture line and eventually reinforce or repair it with sutures [14] or clips. Flexible endoscopy is the best tool as it allows free movement and the exploration of the proximal bowel, as well. In our opinion, rigid sigmoidoscopy has no role.

In conclusion, as with every surgical procedure, the STARR and the $\mathrm{SH}$ also bear risks of postoperative complications. Viewing an effective and established technique negatively only on the basis of surgical complications, which are fortunately rare, is pointless. The surgeons performing these procedures should be aware of the potential for serious adverse events and be ready to deal with them. Pelvic bleeding is one of those unlikely, but potentially lifethreatening, complications that should be identified and treated in a timely manner. An arterial phase CT scan is mandatory to rule out ongoing bleeding and to identify its source. Laparoscopy is the best initial operative approach to have a visual look at the pelvic hematoma and, mostly, to rule out intra-abdominal injuries. Transanal examination and flexible sigmoidoscopy should always be performed to check the intraluminal side of the suture. In the absence of sepsis, no indication exists for a more invasive treatment with an anterior resection and/or fecal diversion, and a demonstrated active bleeding should be treated with arterial embolization, if available. We would suggest not opening the pelvic peritoneum to drain a noninfected pelvic hematoma.

\section{CONFLICT OF INTEREST}

No potential conflict of interest relevant to this article was reported.

\section{REFERENCES}

1. Longo A. Treatment of haemorrhoidal disease by reduction of mucosa and haemorrhoidal prolapse with a circular suturing device: a new procedure. In: Proceedings of the 6th World Congress of Endoscopic Surgery: 6th International Congress of European Association for Endoscopic Surgery (EAES); 199831 May-6 June; Rome, Italy. Bologna: Monduzzi Editore, International Proceedings Division; 1998. p. 777-84,

2. Corman ML, Carriero A, Hager T, Herold A, Jayne DG, Lehur
$\mathrm{PA}$, et al. Consensus conference on the stapled transanal rectal resection (STARR) for disordered defaecation. Colorectal Dis 2006;8:98-101.

3. National Institute for Health and Care Excellence. Circular stapled haemorrhoidectomy: Understanding NICE guidance - information for people considering the procedure, and for the public. NICE Interventional Procedure Guidance IPG34. Dec 2003 [Internet]. London: National Institute for Health and Care Excellence; 2003 [cited $2016 \mathrm{Jul}$ 15]. Available from: https://www.nice. org.uk/guidance/ipg34/resources/circular-stapled-haemorrhoidectomy-255450637.

4. Schwandner O, Stuto A, Jayne D, Lenisa L, Pigot F, Tuech JJ, et al. Decision-making algorithm for the STARR procedure in obstructed defecation syndrome: position statement of the group of STARR Pioneers. Surg Innov 2008;15:105-9.

5. Naldini G. Serious unconventional complications of surgery with stapler for haemorrhoidal prolapse and obstructed defaecation because of rectocoele and rectal intussusception. Colorectal Dis 2011;13:323-7.

6. Pescatori M, Gagliardi G. Postoperative complications after procedure for prolapsed hemorrhoids (PPH) and stapled transanal rectal resection (STARR) procedures. Tech Coloproctol 2008;12: 7-19.

7. Simillis C, Thoukididou SN, Slesser AA, Rasheed S, Tan E, Tekkis PP. Systematic review and network meta-analysis comparing clinical outcomes and effectiveness of surgical treatments for haemorrhoids. Br J Surg 2015;102:1603-18.

8. Jayne DG, Schwandner O, Stuto A. Stapled transanal rectal resection for obstructed defecation syndrome: one-year results of the European STARR Registry. Dis Colon Rectum 2009;52:1205-12.

9. Stuto A, Renzi A, Carriero A, Gabrielli F, Gianfreda V, Villani RD, et al. Stapled trans-anal rectal resection (STARR) in the surgical treatment of the obstructed defecation syndrome: results of STARR Italian Registry. Surg Innov 2011;18:248-53.

10. Schwandner O, Fürst A; German STARR Registry Study Group. Assessing the safety, effectiveness, and quality of life after the STARR procedure for obstructed defecation: results of the German STARR registry. Langenbecks Arch Surg 2010;395:505-13.

11. Meyer P, Stieger R. Retroperitoneal hematoma due to seam insufficiency after stapled hemorrhoidectomy. Chirurg 2004;75:11257.

12. Grau LA, Budó AH, Fantova MJ, Sala XS. Perirectal haematoma and hypovolaemic shock after rectal stapled mucosectomy for haemorrhoids. Int J Colorectal Dis 2005;20:471-2.

13. Blouhos K, Vasiliadis K, Tsalis K, Botsios D, Vrakas X. Uncontrollable intra-abdominal bleeding necessitating low anterior resection of the rectum after stapled hemorrhoidopexy: report of a case. Surg Today 2007;37:254-7.

14. Cerullo G, Cassini D, Martellucci J, Baldazzi G. Laparoscopic approach in a case of retroperitoneal and mesorectal haematoma following STARR procedure. Int J Surg Case Rep 2015;6C:237-40. 